\title{
Eating History Affects Food Palatability in Young Japanese Females
}

\author{
Rie Horiuchi ${ }^{1, *}$, Ram B. Singh ${ }^{2}$, Fabien De Meester ${ }^{3}$, Douglas W. Wilson ${ }^{4}$, Lekh R Juneja ${ }^{5}$ and \\ Toru Takahashi ${ }^{6}$
}

\author{
${ }^{1}$ Department of Food Sciences and Nutrition, Faculty of Human Environmental Sciences, Mukogawa Women's \\ University, Japan \\ ${ }^{2}$ Halberg Hospital and Research Institute, Moradabad, India \\ ${ }^{3}$ The Tsim Tsoum Institute, Krakow, Poland \\ ${ }^{4}$ School of Medicine, Pharmacy and Health, Durham University, UK \\ ${ }^{5}$ Executive Vice President, Taiyo Kagaku Co., Japan \\ ${ }^{6}$ Graduate School of Human Environment Science, Fukuoka Women's University, Japan
}

\begin{abstract}
The purpose of the present study was to elucidate the transition from eating history to food palatability associated with diet selection in adults. Questionnaires were used to identify dominant and favorite recipes, flavorings, and breakfasts for 174 female university students. The participants' recollections for answering the questionnaire covered a six-stage time period: kindergarten, early elementary school years, late elementary school years, junior high school, high school, and the university years. The development of taste and diet choices at the university stage appeared to be influenced by the childhood diet during the kindergarten stage. Controlling food palatability in adults would change diet selection by promoting healthier eating habits, which may decrease the prevalence of noncommunicable diseases.
\end{abstract}

Keywords: Correspondence analysis, Diet selection, Eating history, Food palatability, Younger females.

\section{INTRODUCTION}

There has been a marked increase in our knowledge of the role of diet selection in the global dimensions of noncommunicable diseases [1]. The principal risk factors for noncommunicable diseases vary widely depending on the country. However, poor diet selection by both the poor and wealthy, the double burden of undernutrition and overnutrition, and the triple burden of undernutrition, overnutrition, and sedentary behavior are major risk factors for noncommunicable diseases [2]. Among such risk factors, diet selection, often linked to undernutrition and overnutrition, is controllable, at least theoretically. However, it is difficult to control diet selection, because food palatability does not change radically.

In this study, we hypothesized that food palatability and the dominant diet in childhood may be associated with those as an adult. Generally, food palatability develops in childhood; tastes and diet preferences are learned [3]. Accordingly, food palatability in adults may be affected by the eating history during childhood [4]. A previous study using rats showed an association between food palatability and eating

*Address correspondence to this author at the Department of Food Sciences and Nutrition, Faculty of Human Environmental Sciences, Mukogawa Women's University, 6-46 Ikebiraki-cho, Nishinomiya city, Hyogo prefecture, 663-8558 Japan; Tel: +81-0798-45-3769; Fax: +81-0798-45-3769;

E-mail: mhor9496@mukogawa-u.ac.jp history [5]; the addition of fat to the diets of juvenile rats increased their palatability for fat, even in the adult stages. A few studies attempted to estimate the association between food palatability and eating history [6-9]; however, to date, this association has yet to be determined in humans.

The purpose of the present study was to understand the transition in food palatability that occurs over the course of a person's eating history [10-12]. Diet and health promotion in schools is also discussed.

\section{MATERIALS AND METHODS}

\section{Subjects}

Food palatability for females is different to that of males [13]. We focused on the transition of food palatability in females to restrict gender differences. A total of 210 female, fourth-grade undergraduate students from the Department of Food Science and Nutrition, Mukogawa Women's University, Nishinomiya City, Hyogo Prefecture, Japan, participated in the study. Accordingly, the subjects had extensive knowledge of diet and proper nutrition. The mean age of the subjects was $21.2 \pm 0.4$ (standard deviation (SD)) years. Due to the relatively young age of the subjects, we assumed that they could readily recall their childhood diets. The average height and weight of the subjects was $1.58 \pm 0.05$ (SD) $\mathrm{m}$ and $50.8 \pm 5.6(\mathrm{SD}) \mathrm{kg}$, respectively. A body mass index (BMI) of less than 18.5 was determined for $19.5 \%$ of the 
participants; $75.9 \%$ had a BMI $\left(\mathrm{kg} / \mathrm{m}^{2}\right)$ between 18.5 and 23.5; and 4.6\% presented a BMI of more than 23.5. Most of the subjects were from Osaka (37.4\%) and Hyogo (34.5\%) prefectures; other prefectures represented included Kyoto, Nara, Wakayama, and Siga. Participants resided with their parents $(77.0 \%)$, in an apartment $(20.1 \%)$, or in a dormitory at the university $(1.7 \%)$.

Transitions in food palatability and eating history were estimated from the participants' recollections of childhood diets during the kindergarten (3-6 years old), early elementary school (6-9 years old), late elementary school (9-12 years old), junior high school (12-15 years old), high school (15-18 years old), and university years (18-22 years old), using a self-completed questionnaire. Subjects were surveyed using self-completed questionnaires from April 2011 to September 2011 at Mukogawa Women's University. A total of 210 students from the Department of Food Science and Nutrition at Mukogawa Women's University participated. However, 36 students were absent from class on the day of our assessments. Accordingly, 174 participants answered the questionnaire in its entirety. This study was reviewed and approved by the Fukuoka Women's University Ethics Committee (No: 2010-08).

\section{Questionnaire}

Using a self-completed questionnaire, we assessed the dominant and favorite recipes, favorite flavorings, and dominant breakfasts during six life stages: kindergarten, early elementary school, late elementary school, junior high school, high school, and university years (Table 1). The dominant main dish recipes were those that were cooked regularly. The favorite main dish recipes were those that the participants liked to cook. Of the original 210 participants, 174 subjects completed the questionnaire, a collection rate of $83 \%$. Multiple answers were not allowed. Items on the questionnaire included dominant and favorite recipes, favorite flavorings, and dominant breakfasts.

Favorite recipes and flavorings were used as indices of food palatability. Dominant recipes and breakfasts were used as indices of eating history. A previous study included the following options for dominant and favorite recipes: baked, steamed, sautéd, simmered, boiled, fried, and raw [14]; favorite flavorings options included soy sauce, consommé, mayonnaise, salt, hot chili, garlic, American cheese (cheese), miso, curry, ketchup, Japanese sauce, bechamel sauce, vinegar, sesame, and others. Note that miso is a fermented food, made from soy, rice, yeast, and salt; and Japanese sauce is made from a mixture of vegetables and fruit juice [15].

In this study, we adjusted the favorite flavoring options as follows. Given that Japanese prefer the flavor of soy sauce with sugar to soy sauce alone [16], soy sauce with sugar was added to the favorite flavoring options, and soy sauce was removed. We also added ponzu (a mixture of citrus fruits and soy sauce), demi-glace sauce, bonito soup stock, and Japanese horseradish and mustard (a common Japanese seasoning) [15]. We deleted consommé, hot chili, garlic, and sesame used in the previous study, because these flavorings are not used as frequently in Japanese cooking [17]. Thus, the favorite flavoring options for our study were as follows: soy sauce with sugar, mayonnaise, salt, cheese, miso, curry (a type of curry roux), ketchup, Japanese sauce, bechamel sauce, vinegar, ponzu, demi-glace sauce, bonito soup stock, Japanese horseradish, mustard, and others.

The diet composition usually depends on the staple food, which affects the side dishes $[13,18]$. Thus, in this study, we focused on the staple foods used for breakfast. The dominant breakfast items included rice, white bread, butter rolls, sweet buns, and cornflakes. The Annual Census on Households by the General Affairs Office classifies bread into two groups: sweet buns and white bread (which includes the butter roll) [19]. Sweet buns included sweet croissants, bread covered with sweet topping, and breads with custard cream or sweet beans. Sweet buns, white bread, and butter rolls are consumed frequently in the Japanese diet [19]. However, butter rolls are not the same as white bread; the fat content of the butter roll is twice that of white bread $[10,20,21]$. Thus, butter roll was added to the option list. Cereal is also consumed at a high frequency, with the most common breakfast cereal being cornflakes [22]. As such, cornflakes were added to the option list of dominant breakfast items.

\section{Statistics}

The transition of dominant and favorite recipes, favorite flavorings, and dominant breakfasts, according to life stages, was subjected to correspondence analysis [23]. All statistical analysis was performed using the SPSS version 20.0 (IBM) Base and Categories software. The results were expressed as mean values and SD. Differences with $p<0.05$ were considered significant.

Table 1. Options and items on the questionnaire

\begin{tabular}{|l|l|}
\hline \multicolumn{1}{|c|}{ Options } & \multicolumn{1}{|c|}{ Items } \\
\hline \hline $\begin{array}{l}\text { Which main dish recipe did you usually eat in kindergarten, younger in } \\
\text { elementary school, older in elementary school, in junior high school, in high } \\
\text { school, and as a university student? }\end{array}$ & Baked, steamed, sautéd, simmered, boiled, fried, and raw foods \\
\hline $\begin{array}{l}\text { Which main dish did you like in kindergarten, younger in elementary } \\
\text { school, older in elementary school, in junior high school, in high school, } \\
\text { and as a university student? }\end{array}$ & Baked, steamed, sautéd, simmered, boiled, fried, and raw foods \\
\hline $\begin{array}{l}\text { Which main dish flavoring did you usually eat in kindergarten, younger in } \\
\text { elementary school, older in elementary school, in junior high school, in high } \\
\text { school, and as a university student? }\end{array}$ & $\begin{array}{l}\text { Soy sauce with sugar, mayonnaise, salt, cheese, miso, curry (curry roux), } \\
\text { bonito soup stock, Japanese horseradish, mustard, and others }\end{array}$ \\
\hline
\end{tabular}




\section{RESULTS}

\section{Dominant Recipes and Life Stages}

Fig. (1). shows the interrelationship between dominant recipes and life stages using correspondence analysis. Correspondence analysis indicates the degree of association between two symbols as distance on a graph. For this purpose, new axes were prepared and called the first and second dimensions in the correspondence analysis. The meaning of the dimensions is often abstract. The contributing ratio of the first dimension on the abscissa was 0.744; thus, the firstdimension can explain $74.4 \%$ of the variations. The contributing ratio of the second dimension on the vertical axis was 0.170 , which explains $17.0 \%$ of the variation $(p<0.001)$. The distance between the life stage and dominant recipe symbols indicates the degree of association between the recipe and the life stage of the subject (Fig. 1) [23]. If the distance between a recipe symbol and that of a life stage is short, then the dish was frequently eaten during that particular life stage [23]. The symbols for kindergarten, younger elementary school grades, and older elementary school grades were located close to the symbols for boiled and simmered, baked, and baked and sautéd foods, respectively (Fig. 1). The symbols for junior high school, high school, and undergraduate stages were located close to those of sautéd and fried, sautéd and raw, and raw and boiled and simmered foods, respectively (Fig. 1).

\section{Favorite Recipes and Life Stages}

Fig. (2) shows the correspondence between favorite recipes and life stages. The contributing ratio of the first dimension on the abscissa was 0.725 , and that of the seconddimension on the vertical axis was 0.229 (Fig. $2, p<0.001$ ). The symbols for kindergarten, younger elementary school grades, and older elementary school grades were located close to those of boiled and simmered, baked, and sautéd foods, respectively (Fig. 2). The symbols for junior high school, high school, and university stages were located close to those of fried, sautéd and steamed, and raw and simmered foods, respectively (Fig. 2).

\section{Favorite Flavoring and Life Stages}

Fig. (3) shows the interrelationships between favorite flavorings and life stages. The contributing ratio of the first dimension of the abscissa was 0.704 , and that of the seconddimension on the vertical axis was 0.208 (Fig. 3, $p<0.001$ ). The symbols for kindergarten, younger elementary school grades, older elementary school grades stages were located close to those of ketchup, curry (a type of curry roux), and those of both Japanese sauce and mayonnaise, respectively. The symbols for junior high school, high school, and university stages were located close to all those of salt, bechamel sauce and Japanese sauce, demi-glace sauce, soy sauce with sugar, those of both cheese and bechamel sauce, vinegar,

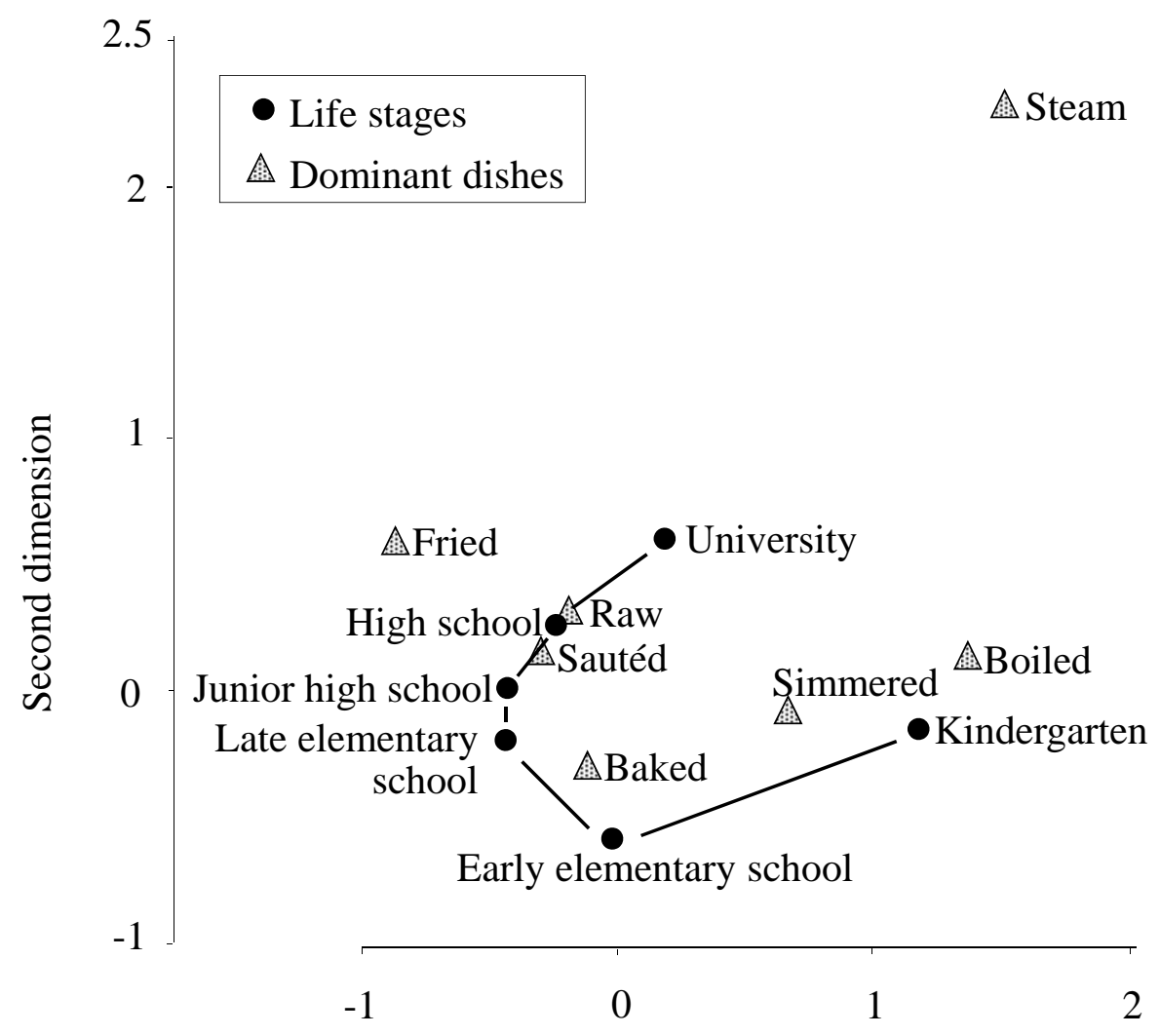

First dimension

Fig. (1). Associations between dominant recipes and life stages. The contributing ratio of the first dimension on the abscissa was 0.744 , indicating that the first dimension can explain $74.4 \%$ of the variations (correspondence analysis). The contributing ratio of the second dimension on the vertical axis was 0.170 ; thus, the second dimension can explain $17.0 \%$ of the variations $(p<0.001) . \mathrm{n}=174$. 


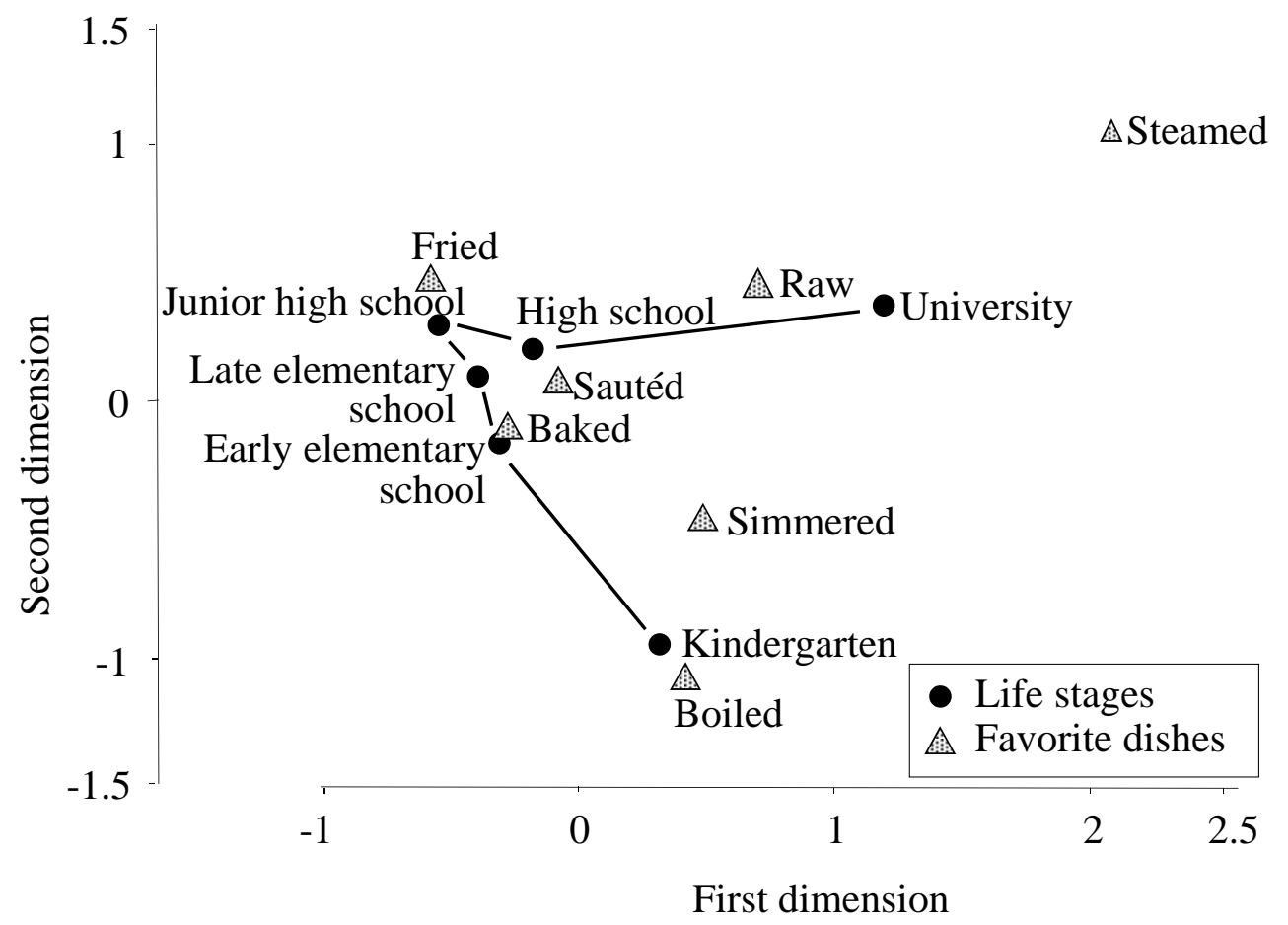

Fig. (2). Associations between favorite recipes and life stages. The contributing ratio of the first dimension on the abscissa was 0.725 (72.5\% of the variation was explained by the first dimension. The contributing ratio of the second dimension on the vertical axis was 0.229 (22.9\% of the variation was explained by the second dimension $(p<0.001)) . \mathrm{n}=174$.

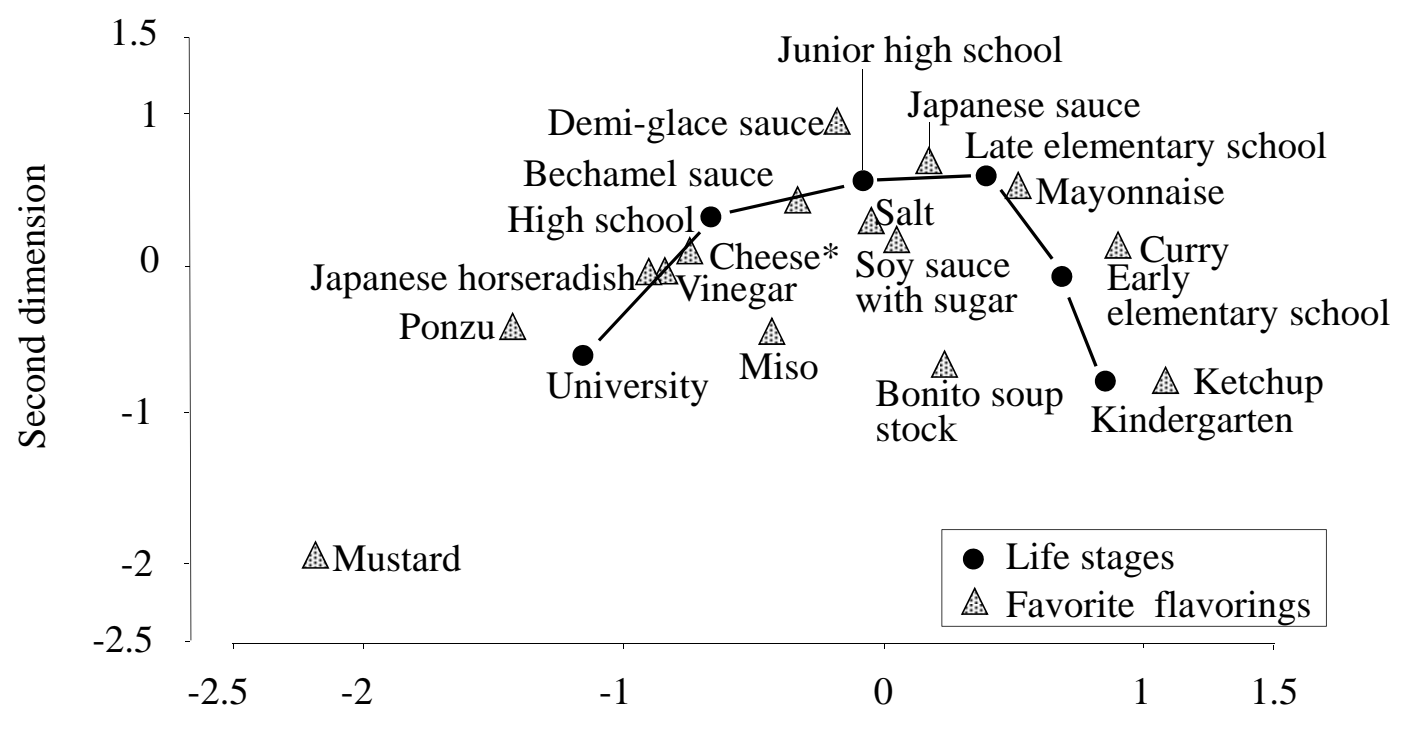

First dimension

Fig. (3). Associations between favorite flavorings and life stages. The contributing ratio of the first dimension on the abscissa was 0.704 ( $70.4 \%$ of the variation). The contributing ratio of the second dimension on the vertical axis was $0.208(20.8 \%$ of the variation $(p<0.001)$. $\mathrm{n}$ $=174 . *$ American cheese.

Japanese horseradish and all those of ponzu, vinegar, Japanese horseradish, and miso, respectively (Fig. 3).

\section{Dominant Breakfast}

Fig. (4) shows the correspondence between dominant breakfast and life stages. The contributing ratio of the first dimension on the abscissa was 0.637 , and that of the second dimension on the vertical axis was 0.257 (Fig. 4, $p<0.001$ ). Kindergarten, younger elementary school grades, and older elementary school grade life stages were located close to the symbols for rice and butter rolls, rice and white bread, and white bread, respectively (Fig. 4). Junior high school, high 


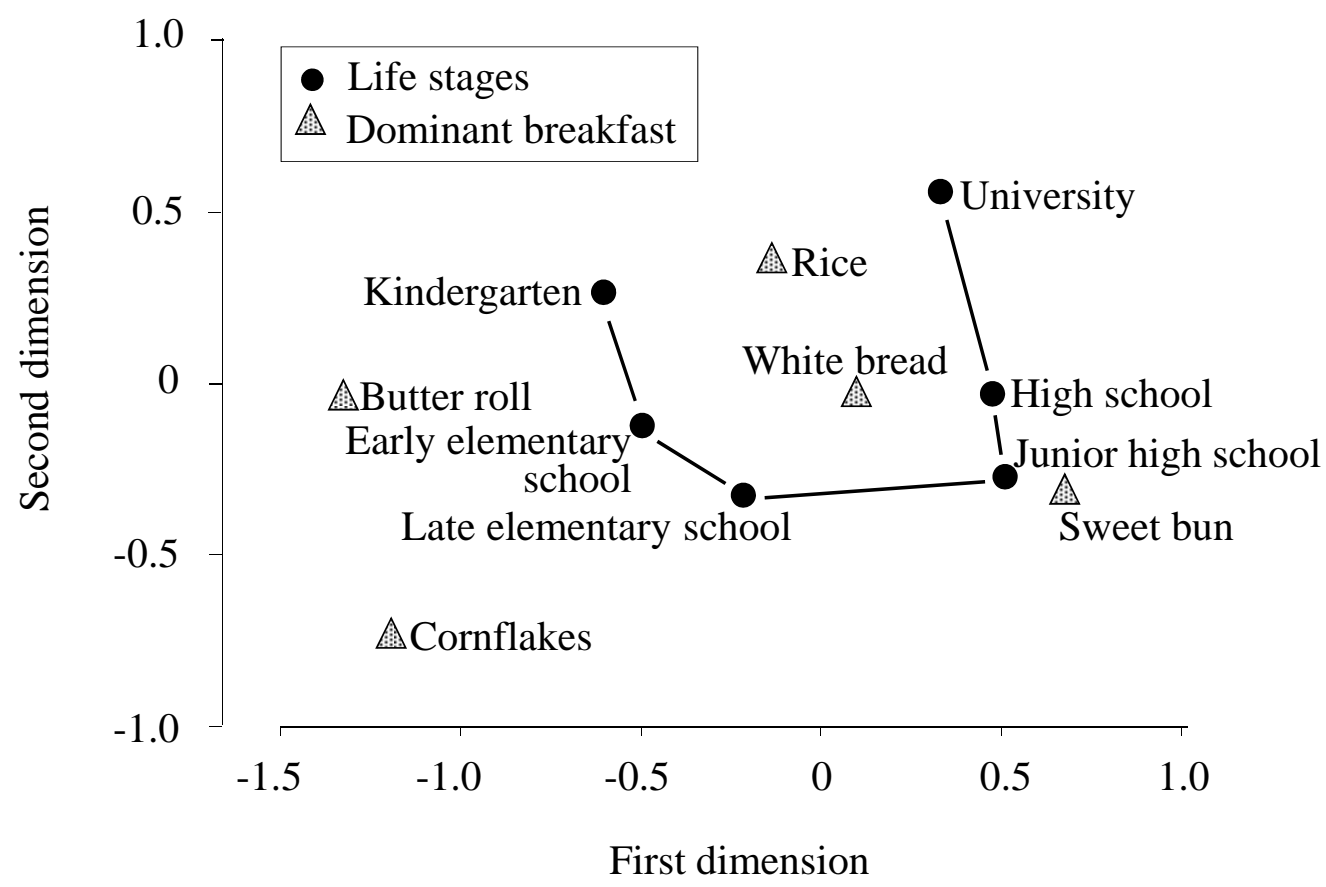

Fig. (4). Associations between dominant breakfast and life stages. The contributing ratio of the first dimension of the abscissa was 0.637 (63.7\% of the variation). The contributing ratio of the second dimension on the vertical axis was $0.257(25.7 \%$ of the variation $(p<0.001))$. $\mathrm{n}$ $=174$.

school, and university life stages were located close to those of sweet buns, rice and white bread, and white bread, respectively (Fig. 4).

\section{DISCUSSION}

The first and second dimensions on the panels in Figs. (1 and 2) could be attributed to the amount of oil in the dishes and the amount of work required to prepare the dishes. The amount of lipids in the dominant and favorite recipes increased from the kindergartner years through the younger grades of the elementary school stage; after the junior high school stage, the amount of lipids decreased as the life stage progressed (Figs. 1 and 2). The level of perception of rich taste increased with developing grades until the lower grades of the elementary school stage, and decreased with increasing grades after the junior high school stage (Fig. 3). The transitions of the dominant recipes, favorite recipes, favorite flavoring, and dominant breakfast showed similar U-shaped curves, with terminals observed at the kindergarten and university stages (Figs. 1-4). Favorite recipes and favorite flavorings were used as indices of food palatability; dominant recipes and dominant breakfasts were indices of eating history. Food palatability is affected by eating history [4]. The eating history in the kindergarten stage appeared to be associated with the food palatability at the university stage, according to the results of our study. This is consistent with our hypothesis, i.e., that food palatability and dominant diets in childhood are associated with those in adulthood.

Figs. (1-4) show similar U-shaped curves in the transitions of eating history (dominant recipes and dominant breakfast) and food palatability (favorite recipes and favorite flavorings); this similar transition pattern was attributed to basic learning and memory phenomena [3]. Learning and memory for dominant diets would increase the palatability of the dominant food in childhood [3].

The effect of eating history on food palatability suggests a method of controlling food palatability in adults. Food palatability in the university or older life stages could be controlled by the dominant diet in the kindergarten stage. The major cause of death in high-income countries is noncommunicable disease [24]. The dominant risk factor for death due to noncommunicable diseases is diet [1]. Thus, careful control and monitoring of the diet in the kindergarten stage may help to control food palatability in adults and may decrease the likelihood of developing a noncommunicable disease. In this case, public health officials should emphasize and provide supplementary education on the importance of a proper diet in the kindergarten years.

Additionally, the traditional food of the local district should be a major component of the diet at the kindergarten stage. Frequent contact with traditional foods eaten in the kindergarten years may increase the palatability of traditional foods when the children become adults. Normally, traditional food is consumed in the home [25]. Home cooking is thus important to facilitate the acceptance of traditional food as the main component of the diet.

Studies have shown that junior high and high school students enjoy the Western diet [14, 26], consistent with our favorite dishes and flavorings results shown in Figs. (2 and 
3). It is difficult to control the diets of students in the junior and high school years, because their preference for foods with high oil and protein content favors Western food [10]. Thus, it may be easier to control the diet in the kindergarten years.

Similarities in the dominant and favorite recipes, favorite flavorings, and breakfasts between the kindergarten and university stages (Figs. 1-3) emphasize that habituation to a prudent diet in the junior high and high school years may not provide the basis needed to control the diet as an adult.

Parents or caregivers determine the diets of children at the kindergarten stage [27]. The effects of parents or caregivers are likely to decrease in high school because of TV and friends [28]. However, food preferences in adults are still affected by parents and caregivers through learning during the kindergarten stage. The effects of parents or caregivers on children do not disappear in later life.

Our study was limited by location and gender. The participants in this study were female university students in the Hyougo prefecture. As such, a similar study with male students may further confirm the relationship between eating history and food palatability.

The data in the questionnaires were based on the students' recollection of their taste for food over a period of time, ranging from kindergarten through the university years; this time period was divided into six life stages. Answering the questionnaire by recollection of childhood eating experiences may have limited the reliability of the data. Previous studies that assessed staple foods for breakfast [26] and dominant dishes at the kindergarten stage [29] showed a high frequency of rice and white bread, and baked and boiled food preparation methods; this is in agreement with our results (Figs. 1 and 4), and supports the validity of the assessment technique used in the present study.

In conclusion, our results showed that food palatability in adults was controlled by the dominant diet in the childhood kindergarten stage. Controlling food palatability in adults by serving a dominant diet in the kindergarten years would facilitate more prudent diet selection as an adult. This, in turn, would promote a healthier diet as an adult and possibly minimize the risk of developing a non communicable disease.

\section{CONFLICT OF INTEREST}

The authors confirm that this article content has no conflicts of interest.

\section{ACKNOWLEDGEMENTS}

This study was supported by a Grant-in-Aid from the Japan Society Food Service and Management program. The author wishes to thank students from the fourth-grade undergraduate program in the Department of Food Science and Nutrition, Mukogawa Women's University. The authors declare no conflict of interest.

\section{REFERENCES}

[1] Tokunaga M, Takahashi T, Singh RB, et al. Diet, nutrients and noncommunicable disease. Open Nutra J 2012; 5: 146-59.
[2] Singh RB, Pella D, Mechirova V, et al. Prevalence of obesity, physical inactivity and undernutrition, a triple burden of diseases during transition in a developing economy. The Five City Study Group. Acta Cardiol 2007; 62(2): 119-27.

[3] Yamamoto T, Ueji K. Brain mechanisms of flavor learning. Frontier Syst Neurosci 2011; 5: 76. doi: 10.3389/fnsys.2011.00076.

[4] Bermúdez-Rattoni F, Núñez-Jaramillo L, Balderas I. Neurobiology of taste-recognition memory formation. Chem Sens 2005; 30(suppl 1): i156-7.

[5] Nakashima Y. Effect of feeding history of fat and cellulose during growth period on preferential fat intake in adult rats. J Jpn Soc Nutr Food Sci 2006; 59: 297-304.

[6] Otsuka R, Tamakoshi K, Shimokata H, Toyoshima H, Yastsuya H. Dietary habits and incidence of metabolic syndrome among middle-aged Japanese male workers. J Jpn Soc Nutr Food Sci 2009; 62(3): 123-9.

[7] Koda T, Ando M, Takasugi M, et al. Relationship between the preference for soup stock perception of umami taste odor among university students. J Home Econom Jpn 2011; 62(3): 179-88.

[8] Shigaki H, Ikeuti M, Konishi F, Hanasaki N. Fish-eating preference and dietary choice among students. J Cook Sci Jpn 2004; 37(2): 206-14.

[9] Kawasaki H, Kanamori Y, Fushiki T. Effect of high-carbohydrate diet flavored with bonito bouillon's on high-fat intake. J Jpn Soc Nutr Food Sci 2002; 55(2): 79-84.

[10] Dietary habit data book in students in elementary and junior high school in 2002. National Agency for the Advancement of Sports and Health: Japan 2001, pp. 54-5.

[11] Drewnowski A. Taste preferences and food intake. Ann Rev Nutr 1997; 17: 237-53.

[12] Wardle J, Sanderson S, Gibson EL, Rapoport L. Factor-analytic structure of food preferences in four-year-old children in the UK. Appetite 2001; 37(3): 217-23.

[13] Fushiki T. Taste and food palatability. Tokyo, Japan: Domesu Press: 2006, 145.

[14] Yukawa H. Longitudinal study on dietary intake by the elderly in urban community. Jpn J Nutr Dietetic 2004; 62(2): 73-81.

[15] Digital Dai-ji-sen Japanese Dictionary, Tokyo, Japan: Shogakukan, Inc. 2012: p. 2.

[16] Kojo A, Takagi R. Food preference of elderly patients in a health care facility. Jpn J Nutr Diet 2004; 62: 153-60.

[17] Fukuda S, Ikeda J. Transition in breakfast meals Based on the intakes by female college students. Bull Kyoto Bunkyou Junior Coll 2007; 46: 38-46.

[18] Harigaya J, Izumi T. The review of an appropriate amount for one meal based on bread and noodles as staple food: expanding $3 \bullet 1$ - 2 the lunch box dietary method to a different type of staple food. Bulletin of the Faculty of Education, Kouchi University 2007; vol. 67: pp. 69-79.

[19] Ohmori R, Yamazaki H, Iida Y. Iwahara Y, Nagayama K. The survey of dietary habits in preschool children. Bull Utunomiya Univ 2007; 30: 361-8.

[20] Goto Y, Oishi N, Takenaka R, Furukawa K. A survey on university students' breakfast: Focusing on their dietary behavior. Bull Yamaguchi Junior Coll 2003; 53: 31-50.

[21] Etoh H, Kitano N, Minami H. Survey on lunch program and food habits for children and guardian by kindergartens at Kumamoto. J Integrat Study Diet Habit 2009; 20(3): 195-202.

[22] Ayabe S, Konishi F, Otsuka E. Infants' Breakfast Eating Habits and Parents’ Dietary Awareness. Jpn J Nutr Diet 2005; 63: 273-83. Ishikawa S. Multivariate analysis for identifying English basic words. Application of principal component analysis, factor analysis, and correspondence analysis. Inst Statis Math 2007; 199: 2748.

[24] The PURE Investigators. Prevalence of a healthy lifestyle among individuals with cardiovascular disease in high-, middle- and lowincome countries: the prospective urban rural epidemiology (PURE) study. JAMA 2013; 309: 1613-21.

[25] Kosuge M, Fuseya S, Nakajima A, et al. Transmission and prospect of culture in life style in three generation families. Bull Wayo Women's Univ 2001; 41: 97-106.

[26] Seki C, Kato E, Narita T. The dietary condition of preschool children: dietary intake of children and dietary consciousness of parents. Bull Shukutoku Junior Coll 2003; 42: 127-40. 
[27] Orlet Fisher J, MS, Birch L. Fat preferences and fat consumption of 3- to 5-year-old children are related to parental adiposity. J Am Diet Assoc 1995; 95(7): 759-64.
[28] Wardle J, Cooke L. Genetic and environmental determinants of children's food preferences. Br J Nutr 2008; 99: 15-21.

[29] Gámiz F, Gallo M. Taste learning and memory: A window on the study of brain aging. Front Syst Neurosci 2011; 5: 91.

(C) Horiuchi et al.; Licensee Bentham Open.

This is an open access article licensed under the terms of the Creative Commons Attribution Non-Commercial License (http://creativecommons.org/licenses/by-nc/3.0/) which permits unrestricted, non-commercial use, distribution and reproduction in any medium, provided the work is properly cited. 